\title{
On the Stability of Modified Friedrichs Scheme for the Mixed Problem for Symmetric Hyperbolic System
}

\author{
By \\ Yoshinori KAMETAKA*
}

\section{Introduction}

It is well known that the well posed Cauchy problem admits the consistent stable finite difference scheme. By the Lax's equivalence theorem the solutions of that scheme converge to the true solutions of the Cauchy problem.

In the case of mixed problem these general theory is not yet established. Especially in the case of $m$-space variable we can find no example of consistent stable finite difference scheme. But we can say in the case of well posed mixed problem consistency and stability assures $L^{2}$ convergence of approximate solutions to the true solution. Many authors obtained the condition for the stability of finite difference scheme with boundary condition (Strang [1], Kreiss [2], Osher [3]). But they did not find consistent stable scheme which approximate well posed mixed problem for hyperbolic system.

We consider in a half space first order symmetric hyperbolic system. On the plane boundary we set dissipative boundary condition. In the interior of the region we must take the consistent scheme which is stable for the Cauchy problem. Here we consider a modified Friedrichs scheme as a most simple explicit scheme which is stable for the Cauchy problem. We can find consistent boundary scheme which assures the stability of the whole scheme.

Received May 5, 1968.

Communicated by S. Matsuura.

* Osaka City University. 


\section{Symmetric Hyperbolic System}

Consider a mixed problem for symmetric hyperbolic system in the half space $R_{+}^{m}$

$$
\begin{gathered}
\frac{\partial u}{\partial t}=D(x, y) \frac{\partial u}{\partial x}+F_{1}(x, y) \frac{\partial u}{\partial y_{1}}+\cdots+F_{m-1}(x, y) \frac{\partial u}{\partial y_{m-1}} \\
(t, x, y) \in[0, T] \times[0, \infty) \times R^{m-1} \\
P_{-} B(y) u(t, 0, y)=0 \quad(t, y) \in[0, T] \times R^{m-1} \\
u(0, x, y)=u^{0}(x, y) \quad(x, y) \in[0, \infty) \times R^{m-1} .
\end{gathered}
$$

Assumption 1.

$$
D(0, y)=\left(\begin{array}{lll}
D_{+}(y) & & \\
& D_{-}(y)
\end{array}\right)=\left(\begin{array}{lll}
d_{1}(y) & & \\
& \ddots & \\
& & d_{N}(y)
\end{array}\right)
$$

is a real diagonal $N \times N$ matrix, $d_{1}(y), \cdots, d_{p}(y)>\delta>0>-\delta>d_{p^{+1}}(y), \cdots, d_{N}(y) p+q=N F_{i}(x, y) i=1, \cdots$, $m-1$ are real symmetric $N \times N$ matrices

$$
B(y)=\left(\begin{array}{cc}
I_{p} & 0 \\
b(y) & I_{q}
\end{array}\right) I_{p},
$$

$I_{q}$ are identity matrices of order $p$ and $q$ respectively, $b(y)$ is $q \times p$ real matrix.

$$
P_{+}=\left(\begin{array}{ll}
I_{p} & \\
& 0
\end{array}\right), \quad P_{-}=\left(\begin{array}{ll}
0 & \\
& I_{q}
\end{array}\right) .
$$

Under the assumption 1 the mixed problem (1), (2), (3) is well posed.

As a finite difference approximation to the mixed problem (1), (2), (3) we take the modified Friedrichs scheme

$$
\begin{aligned}
& u^{n+1}(j, k)=\frac{1}{4}\left[u(j+1, k)+u(j-1, k)+\frac{1}{m-1} \sum_{i=1}^{m-1}\left\{u\left(j, k+1_{i}\right)+\right.\right. \\
& \left.+u\left(j, k-1_{i}\right)^{\vdots}\right]+\frac{1}{2} \lambda[D(j, k)\{u(j+1, k)-u(j-1, k)\}+ \\
& \left.+\sum_{i=1}^{m-1} F_{i}(j, k)\left\{u\left(j, k+1_{i}\right)-u\left(j, k-1_{i}\right)\right\}\right] \quad(j \geqq 1)
\end{aligned}
$$


$\left(2^{\prime}\right) \quad u^{n+1}(0, k)=\frac{1}{2} B^{-1}(k) P+\left[\frac{1}{2(m-1)} \sum_{i=1}^{m-1}\left\{u\left(0, k+1_{i}\right)+u\left(0, k-1_{i}\right)\right\}+\right.$

$$
+u(1, k)]
$$

(3') $u^{0}(j, k)=u^{0}(j, k)$.

Here we use the abbreviation $u^{n}(j, k)=u(n \lambda h, j h, k h),(j, k)=\left(j, k_{1}, \cdots\right.$, $\left.k_{m-1}\right) j, k_{i}$ are integers, $1_{i}=(0, \cdots, \stackrel{i}{1}, \cdots, 0), h$ is mesh-width, $\lambda h$ is time-step and $\lambda$ is mesh ratio. This scheme $\left(1^{\prime}\right),\left(2^{\prime}\right),\left(3^{\prime}\right)$ is accurate of order 1 to the mixed problem (1), (2), (3).

\section{Assumption 2.}

$$
\left(B^{-1}(y) P_{+}\right) *\left(B^{-1}(y) P_{+}\right) \leqq I+2 \lambda D(0, y)
$$

that is $b^{*}(y) b(y) \leqq 2 \lambda D_{+}(y), I_{q}+2 \lambda D_{-}(y) \geqq 0$.

Assumption 3.

$$
\lambda \sup _{\substack{|\eta|=1 \\(x, y)}}|| F(x, y) \cdot \eta\left\|=\frac{1}{\sqrt{5(m-1)}} \quad \lambda \sup _{\substack{(x, y) \\(x)}}\right\| D(x, y) \| \leqq \frac{1}{2}
$$

\section{Theorem 1.}

Under the assumption 1, 2, 3 the modified Friedrichs scheme $\left(1^{\prime}\right),\left(2^{\prime}\right),\left(3^{\prime}\right)$ is stable, and the solution of $\left(1^{\prime}\right),\left(2^{\prime}\right),\left(3^{\prime}\right)$ satisfies following energy inequality

$$
\left\|u^{n}\right\|_{L^{2}\left(R_{+}^{m}\right)} \leqq \text { const. }\left\|u^{0}\right\|_{L^{2}\left(R_{+}^{m}\right)} .
$$

In the constant coefficient case we may take the constant in the above inequality equal to 1 . Here

$$
\|u\|_{L^{2}\left(R_{+}^{m}\right)}^{2}=h^{m}\left[\frac{1}{2} \sum_{k}|u(0, k)|^{2}+\sum_{\substack{j \geq 1 \\ k}}|u(j, k)|^{2}\right] .
$$

\section{Remark.}

To clarify the meaning of the condition in the assumption 2 we shall remark the following fact. In the case of constant coefficients if we take too small value of the mesh ratio $\lambda$, we can construct the solution of the modified Friedrichs scheme which violates the local energy inequality of the form

$$
\left\|\left.u^{1}\right|_{L^{2}\left(R_{+}^{m}\right)} \leq(1+0(h))\right\| u^{0} \|_{L^{2}\left(R_{+}^{m}\right)} .
$$




\section{Proof of Theorem 1}

We shall prove the theorem 1 only in the case of constant coefficient. Proof in the case of variable coefficient is reduced to that of the constant coefficient case by usual technique.

First we shall introduce the discrete Fourier transform of the function which is defined only on the mesh point in the half space $R_{+}^{m}$

$$
\tilde{u}=\tilde{u}(\xi, \eta)=\sum_{j \geq 1} \hat{u}_{j} e^{-i j \xi}, \quad \hat{u}_{j}=\hat{u}_{j}(\eta)=\sum_{k} u(j, k) e^{-i k \eta} .
$$

By the Parseval's equality

$$
\|u\|_{L^{2}\left(R_{+}^{m}\right)}^{2}=\frac{h^{m}}{(2 \pi)^{m-1}}\left[\frac{1}{2}\left|\hat{u}_{0}\right|^{2}+\sum_{j \geqq 1}\left|\hat{u}_{j}\right|^{2}\right] .
$$

Here

$$
\left|\hat{u}_{j}\right|^{2}=\int_{\mid \eta_{i} \backslash \leqq \pi} \hat{u}_{j}(\eta) \cdot \hat{u}_{j}(\eta) d \eta . \quad i=1, \cdots, m-1 .
$$

Let remark following relations

(4) $\sum_{j \geqq 1} \frac{1}{2}\left(\hat{u}_{j+1}+\hat{u}_{j-1}\right) e^{-i j \xi}=\cos \xi \tilde{u}-\frac{1}{2}\left(\hat{u}_{1}-e^{-i \xi} \hat{u}_{0}\right)$
(5) $\sum_{j \geqq 1} \frac{1}{2}\left(\hat{u}_{j+1}-\hat{u}_{j-1}\right) e^{-i j \xi}=i \sin \xi \tilde{u}-\frac{1}{2}\left(\hat{u}_{1}+e^{-i \xi} \hat{u}_{0}\right)$.

Under the discrete Fourier transform with respect to $y$ the modified Friedrichs scheme is written as

(6) $\hat{u}_{j}^{n+1}=\frac{1}{4}\left[\hat{u}_{j+1}+\hat{u}_{j-1}+\frac{2 \sum_{i=1}^{m-1} \cos \eta_{i}}{m-1} \hat{u}_{j}\right]$

$$
+\frac{1}{2} \lambda\left[D\left(\hat{u}_{j+1}-\hat{u}_{j-1}\right)+i F \cdot \sin \eta \hat{u}_{j}\right]=E_{j}+S_{j}
$$

( 7) $\hat{u}_{0}^{n+1}=\frac{1}{2} B^{-1} P_{+}\left[\frac{\sum_{i=1}^{m-1} \cos \eta_{i}}{m-1} \hat{u}_{0}+\hat{u}_{1}\right]$

we abbreviate hereafter $\hat{u}_{j}^{n}$ as $\hat{u}_{j}$. The amplification matrix of $\left(1^{\prime}\right)$ is 
(8) $\quad C=C(\xi, \eta)=\frac{1}{2}\left(\cos \xi+\frac{1}{m-1} \sum_{i=1}^{m-1} \cos \eta_{i}\right)+i \lambda(D \sin \xi+F \cdot \sin \eta)$ $F \cdot \sin \eta=F_{1} \sin \eta_{1}+\cdots+F_{m-1} \sin \eta_{m-1}$

(9) $\quad C * C=\frac{1}{4}\left(\cos \xi+\frac{1}{m-1} \sum_{i=1}^{m-1} \cos \eta_{i}\right)^{2}+\lambda^{2}(D \sin \xi+F \cdot \sin \eta)^{2}$

$$
\begin{aligned}
& \leqq 1-\frac{1}{2} \sin ^{2} \xi-\frac{1}{2(m-1)}|\sin \eta|^{2}+2 \lambda^{2} D^{2} \sin ^{2} \eta+2 \lambda^{2}(F \cdot \sin \eta)^{2} \\
& |\sin \eta|^{2}=\sin ^{2} \eta_{1}+\cdots+\sin ^{2} \eta_{m-1}
\end{aligned}
$$

By Parseval's equality it is sufficient to show

$$
\left(\frac{1}{2}\left|\hat{u}_{0}^{n+1}\right|^{2}+\sum_{j \geq 1}\left|\hat{u}_{j}^{n+1}\right|^{2}\right)-\left(\frac{1}{2}\left|\hat{u}_{0}\right|^{2}+\sum_{j \geq 1}\left|\hat{u}_{j}\right|^{2}\right) \leq 0 \text {. }
$$

Replacing $\hat{u}_{j}^{n+1}$ in (10) by the right hand side member of (6) and (7), and restricting $\lambda$ as assumption 3 we calculate as follows

$$
\begin{aligned}
\left.\sum_{j \geq 1} E_{j}\right|^{2}- & \sum_{j \geq 1}\left|u_{j}\right|^{2} \\
\leq & -\sum_{j \geq 1}\left[\frac{1}{8}\left|\hat{u}_{j+1}-\hat{u}_{j-1}\right|^{2}+\frac{1}{2(m-1)}|\sin \eta|^{2} \hat{u}_{j} \cdot \hat{u}_{j}\right]+\frac{1}{4}\left|\hat{u}_{0}\right|^{2} \\
& -\frac{1}{4}\left|\hat{u}_{1}\right|^{2}
\end{aligned}
$$

(12) $\sum_{j \geq 1}\left|S_{j}\right|^{2}$

$$
\leq \sum_{j \geq 1}\left[\frac{1}{2} \lambda^{2}|| D\left\|^{2}\left|\hat{u}_{j+1}-\hat{u}_{j-1}\right|^{2}+2 \lambda^{2}|| F\right\|_{1}^{2}|\sin \eta|^{2} \hat{u}_{j} \cdot \hat{u}_{j}\right] .
$$

Using relation (4) and (5) and by Parseval's equality

(13) $\sum_{j \geq 1} 2 \operatorname{Re} E_{j} \cdot S_{j}=\frac{1}{2 \pi} 2 \operatorname{Re}\left(\frac{1}{2}\left(\cos \xi+\frac{1}{m-1} \sum_{i=1}^{m-1} \cos \eta_{i}\right) \tilde{u}-\frac{1}{4}\left(\hat{u}_{1}-e^{-i \xi} \hat{u}_{0}\right)\right.$,

$$
\begin{aligned}
& \left.i \lambda(D \sin \xi+F \cdot \sin \eta) \tilde{u}-\frac{1}{2} \lambda D\left(\hat{u}_{1}+e^{-i \xi} \hat{u}_{0}\right)\right)_{L^{2}\left(|\xi| \leq \pi,\left|\eta_{i}\right| \leq \pi\right)} \\
& \quad=-\frac{1}{4} \lambda D \hat{u}_{0} \cdot \hat{u}_{0}-\frac{1}{4} \lambda D \hat{u}_{1} \cdot \hat{u}_{1} \\
& \quad-R e \frac{1}{2(m-1)} \sum_{i=1}^{m-1} \cos \eta_{i} \hat{u}_{1} \cdot \lambda D \hat{u}_{0}+R e \frac{1}{2} \hat{u}_{0} \cdot i \lambda F \cdot \sin \eta \hat{u}_{1} .
\end{aligned}
$$

By the assumption 2 
(14) $\frac{1}{2}\left|\hat{u}_{0}^{n+1}\right|^{2} \leqq \frac{1}{8}(I+2 \lambda D)\left(\frac{1}{m-1} \sum_{i=1}^{m-1} \cos \eta_{i} \hat{u}_{0}+\hat{u}_{1}\right) \cdot$

$$
\begin{aligned}
& \cdot\left(\frac{1}{m-1} \sum_{i=1}^{m-1} \cos \eta_{i} \hat{u}_{0}+\hat{u}_{1}\right) \\
& \quad \leqq \frac{1}{8}(I+2 \lambda D) \hat{u}_{0} \cdot \hat{u}_{0}+\operatorname{Re} \frac{1}{4}(I+2 \lambda D) \frac{1}{m-1} \sum_{i=1}^{m-1} \cos \eta_{i} \hat{u}_{0} \cdot \hat{u}_{1} \\
& \quad+\frac{1}{8}(I+2 \lambda D) \hat{u}_{1} \cdot \hat{u}_{1} .
\end{aligned}
$$

It follows that

$$
\begin{aligned}
& \left(\frac{1}{2}\left|\hat{u}_{0}^{n+1}\right|^{2}+\sum_{j \geq 1}\left|\hat{u}_{j}^{n+1}\right|^{2}\right\rangle-\left(\frac{1}{2}\left|\hat{u}_{0}^{n}\right|^{2}+\sum_{j \geqq 1}\left|\hat{u}_{j}^{n}\right|^{2}\right) \\
& \leqq-\sum_{j \geqq 1} \frac{1}{8}\left(1-4 \lambda^{2}|| D \|^{2}\right)\left|\hat{u}_{j+1}-\hat{u}_{j-1}\right|^{2}- \\
& -\sum_{j \geqq 1} \frac{1}{2(m-1)}\left(1-4(m-1) \lambda^{2}|| F \|^{2}\right)|\sin \eta|^{2} \hat{u}_{j} \cdot \hat{u}_{j}- \\
& -\frac{1}{8}\left|\hat{u}_{0}\right|^{2}-\frac{1}{8}\left|\hat{u}_{1}\right|^{2}+\operatorname{Re} \frac{1}{4(m-1)} \sum_{i=1}^{m-1} \cos \eta_{i} \hat{u}_{0} \cdot \hat{u}_{1}+ \\
& +R e \frac{1}{2} \hat{u}_{0} \cdot i \lambda F \cdot \sin \eta \hat{u}_{1} \\
& \leqq-\frac{1}{8(m-1)} \sum_{i=1}^{m-1}\left(\left|\hat{u}_{0}\right|^{2}+\left|\hat{u}_{1}\right|^{2}-2 \operatorname{Re} \cos \eta_{i} \hat{u}_{0} \cdot \hat{u}_{1}\right)+ \\
& +R e \frac{1}{4(m-1)} \sum_{i=1}^{m-1}\left(2 \hat{u}_{0}-2 \cos \eta_{i} \hat{u}_{1}\right) \cdot i \lambda F \cdot \sin \eta \hat{u}_{1}- \\
& -\frac{1}{2(m-1)}\left(1-4(m-1) \lambda^{2}|| F||^{2}\right)|\sin \eta|^{2} \hat{u}_{1} \cdot \hat{u}_{1} \\
& =-\frac{1}{8(m-1)} \sum_{i=1}^{m-1}\left(\frac{1}{2}\left|\hat{u}_{0}-e^{i \eta_{i}} \hat{u}_{1}\right|^{2}+\frac{1}{2}\left|\hat{u}_{0}-e^{-i \eta_{i}} \hat{u}_{1}\right|^{2}\right)+ \\
& +\frac{1}{4(m-1)} \operatorname{Re} \sum_{i=1}^{m-1}\left\{\left(\hat{u}_{0}-e^{i \eta_{i}} \hat{u}_{1}\right)+\left(\hat{u}_{0}-e^{-i \eta_{i}} \hat{u}_{1}\right)\right\} \cdot \\
& \cdot i \lambda F \cdot \sin \eta \hat{u}_{1}-\frac{1}{2(m-1)}\left(1-4(m-1) \lambda^{2}|| F \|^{2}\right)|\sin \eta|^{2} \hat{u}_{1} \cdot \hat{u} . \\
& \leqq-\frac{1}{2(m-1)}\left(1-\left.5(m-1) \lambda^{2}|| F\right|^{2}\right)|\sin \eta|^{2} \hat{u}_{1} \cdot \hat{u}_{1} \quad \leqq 0 \text {. }
\end{aligned}
$$

This completes the proof of the theorem 1 . 


\section{Non Symmetric Perturbation}

Next we consider the case in which (1) is constant coefficient symmetric and simultaneously regularly hyperbolic outside some compact set $K$ in $R_{+}^{m}$, on the compact set $K(1)$ is variable coefficient and regularly hyperbolic, and on the neighbourhood of the plane boundary of the region $R_{+}^{m}(1)$ is symmetric hyperbolic.

Theorem 2.

In this case theorem 1 is also true. But we must modify the assumption 3 on the non symmetric region $K$ as follows

Assumption $3^{\prime}$.

$$
\lambda \sup _{\substack{1 \leqq j \leqq N \\(x, y) \in K \\ \xi^{2}+|\eta|^{2}=1}}\left|\mu_{j}(x, y, \xi, \eta)\right| \leqq \frac{1}{\sqrt{2(m-1)}} .
$$

Here $\mu_{j}$ is the eigenvalues of

$$
D(x, y) \xi+\sum_{i=1}^{m-1} F_{i}(x, y) \eta_{i} .
$$

\section{Hyperbolic System with One Space Variable}

In this case Friedrichs scheme is as follows

$$
\begin{array}{ll}
\left(1^{\prime \prime}\right) \quad u^{n+1}(j)=\frac{1}{2}\left\{u^{n}(j+1)+u^{n}(j-1)\right\}+\frac{1}{2} \lambda D(j)\left\{u^{n}(j+1)-u^{n}(j-1)\right\} \\
\left(2^{\prime \prime}\right) \quad u^{n+1}(0)=B^{-1} P_{+} u^{n}(1) . \\
\left(3^{\prime \prime}\right) \quad u^{0}(j)=u^{0}(j) \quad(j \geqq 0) .
\end{array}
$$

Assumption 2".

$$
\left(B^{-1} P_{+}\right) * B^{-1} P_{+} \leqq I+\lambda D(0)
$$

Assumption $3^{\prime \prime}$.

$$
\lambda \sup _{x \geqq 0} \| D(x) i \mid \leqq 1
$$

Theorem 3.

Under the assumption $1,2^{\prime \prime}, 3^{\prime \prime}$ the Friedrichs scheme $\left(1^{\prime \prime}\right),\left(2^{\prime \prime}\right)$, 
$\left(3^{\prime \prime}\right)$ is stable and same energy inequality as that of theorem 1 is true.

The author thanks Professor M. Yamaguti for his kind advice and encouragement.

\section{References}

[1] Strang, G., Wiener-Hopf difference equations, J. Math. Mech. 13 (1964), 85-96.

[2] Kreiss, H. O., Difference approximations for the initial-boundary value problem for hyperbolic differential equations, Numerical Solutions of Nonlinear Partial Differential Equations, Wiley, New York (1966), 141-166.

[3] Osher, S., Systems of difference equations with general homogeneous boundary conditions, Brookhaven National Laboratory Report, BNL 11857, Upton, New York, 1968.

[4] Yamaguti, M. and T. Nogi, An algebra of pseudo difference schemes and its application, Publ. RIMS Kyoto Univ. Ser. A, 3 (1967), 151-166. 